\title{
Comparison of the Distribution of Oncotype Dx Recurrence Scores Between Invasive Ductal Carcinoma and Invasive Lobular Carcinoma of the Breast
}

\author{
Sumeet K. Yadav, MD'1, Siddhartha Yadav, MD, FACP ${ }^{2}$ \\ 1 Senior Associate Consultant, Hospital Internal Medicine, Mayo Clinic, Mankato, MN, 2 Assistant Professor, Oncology, Mayo Clinic, Rochester, MN \\ Keywords: oncotype $d x$, invasive ductal carcinoma, invasive lobular carcinoma, breast cancer \\ https://doi.org/10.53876/001c.28398
}

International Journal of Cancer Care and Delivery

Vol. 1, Issue Supplement 1, 2021

\section{INTRODUCTION:}

Oncotype Dx assay has revolutionized breast cancer treatment as a prediction tool for recurrence risk and benefits from adjuvant chemotherapy in estrogen receptor (ER) positive, human epidermal receptor growth factor 2 receptor (HER2) negative breast cancer. Here we compare the distribution of recurrence scores from Oncotype Dx between invasive ductal carcinoma (IDC) and invasive lobular carcinoma (ILC) of the breast.

\section{METHODS:}

The study population included women older than 18 with ER-positive, HER2 negative IDC, and ILC between 2010 to 2016 in the National Cancer Database. The study was restricted to women who had Oncotype Dx test results available. In addition, those with more than three lymph nodes by breast cancer were excluded from the analysis. The distribution of the recurrence scores between ILC and IDC cases was compared utilizing a t-test. All analyses were performed using SPSS version 21.

\section{RESULT:}

A total of 30627 patients met our inclusion criteria, out of which 25979 (84.8\%) were IDC and 4648 (15.2\%) were ILC. Mean age at breast cancer diagnosis was similar between the two groups (59.38 Vs. 60.96 years). The majority of patients with IDC had poorly differentiated tumors when compared to ILC. The mean recurrence score in the IDC group was $17 \pm 9.74$, whereas it was $16 \pm 6.96$ in the ILC group $(\mathrm{p}=0.001)$. The median recurrence score was 16 and 15 in IDC and ILC, respectively. The distribution of recurrence score by specific clinical cutoff was significantly different between IDC and ILC, as demonstrated in Table 1.

\section{CONCLUSION:}

This study demonstrates that patients with IDC have a higher Oncotype Dx score when compared to ILC, which has implications for chemotherapy administration by histological subtype of cancer. 
Table 1. Oncotype Dx recurrence score in IDC and ILC

\begin{tabular}{|l|l|l|}
\hline OncotypeDx Recurrece Score & IDC & ILC \\
\hline $0-11$ & $7408(28.5 \%)$ & $1306(28.1 \%)$ \\
\hline $12-25$ & $14828(57.1 \%)$ & $3047(65.6 \%)$ \\
\hline $26+$ & $3743(14.4 \%)$ & $295(6.3 \%)$ \\
\hline
\end{tabular}

p-value $=0.001$ 